\title{
Infinite 2-class field towers of some imaginary quadratic number fields
}

\author{
by \\ Yutaka Sueyoshi (Nagasaki)
}

1. Introduction. Let $K$ be an imaginary quadratic number field with discriminant $d$, and $C_{K}$ denote the ideal class group of $K$. We mean by the 2class field tower of $K$ the sequence of fields $K=K_{0} \subseteq K_{1} \subseteq \ldots \subseteq K_{i} \subseteq \ldots$, where $K_{i+1}$ is the Hilbert 2-class field (i.e. the maximal unramified abelian 2-extension) of $K_{i}$. If $K_{i+1} \neq K_{i}$ for all $i$, then we say that the 2-class field tower of $K$ is infinite.

By the results of Golod-Shafarevich [3] and Vinberg-Gaschütz [12, 15], the 2-class field tower of $K$ is infinite if 2-rank $C_{K} \geq 5$. On the other hand, Koch [6] and Hajir $[4,5]$ proved that the 2-class field tower of $K$ is infinite if 4-rank $C_{K} \geq 3$. When 2-rank $C_{K}=3$ and 4-rank $C_{K}=0$, there are some examples of infinite families of $K$ with infinite (resp. finite) 2-class field towers $[4,7]$. However, when 2 -rank $C_{K}=4$, no example of $K$ with finite 2-class field tower has ever been known. It has been conjectured [9] that the 2 -class field tower of such a $K$ is always infinite. In this direction, Benjamin $[1,2]$ proved that the 2-class field tower of $K$ is infinite if 2-rank $C_{K}=4$ and 4-rank $C_{K}=2$, except some type of Rédei matrix of $K$.

In this paper, we study the case where 2 -rank $C_{K}=4$ and exactly one negative prime discriminant divides $d$, and prove that the 2-class field tower of such a $K$ is infinite, except for one type of Rédei matrix of $K$. To prove our theorem, we use Martinet's inequalities [9] and their corollaries. We also use some properties of Rédei matrices [10, 11, 13, 14]. A similar problem for real quadratic number fields is treated by Maire [8], by a different method.

2. Martinet's inequalities and their corollaries. Let $K$ be an imaginary quadratic number field.

MARTINET'S inEQUALITY (general case, [9]). Let E/F be a quadratic extension of number fields. Denote by $r_{1}\left(\right.$ resp. $\left.r_{2}\right)$ the number of real (resp.

2000 Mathematics Subject Classification: Primary 11R37; Secondary 11R11, 11R29.

Key words and phrases: quadratic fields, class group, class field tower. 
imaginary) places of $F$. Also denote by $t$ (resp. $\varrho$ ) the number of finite (resp. infinite) places of $F$ which ramify in $E$. If

$$
t \geq r_{1}+r_{2}-\varrho+3+2 \sqrt{2\left(r_{1}+r_{2}\right)-\varrho+1},
$$

then the 2-class field tower of $E$ is infinite.

MARTINET'S INEQUALITY I. Let $F$ be a totally real number field of degree $n$, and $E$ a totally imaginary quadratic extension of $F$. Let $t$ be the number of prime ideals of $F$ which ramify in $E$. If

$$
t \geq 3+2 \sqrt{n+1}
$$

then the 2-class field tower of $E$ is infinite.

Proof. Since $r_{1}=\varrho=n$ and $r_{2}=0$, the assertion follows from the general case of Martinet's inequality.

Corollary 1. Let $F$ be a real quadratic number field. Suppose that four rational primes split in $F$ and ramify in $K$, or that a rational prime remains prime in $F$ and three other rational primes split in $F$ and these four rational primes ramify in $K$. Then the 2 -class field tower of $E=F K$ is infinite.

Proof. Since $n=2$ and $t \geq 7 \geq 3+2 \sqrt{2+1}=6.464 \ldots$ in these cases, the 2-class field tower of $E=F K$ is infinite by Martinet's inequality I.

Corollary 2. Let $F$ be a totally real number field of degree 4. Suppose that two rational primes split completely in $F$ and ramify in $K$, or that a rational prime splits completely in $F$ and two other rational primes are unramified and split into at least two primes in $F$ and these three rational primes ramify in $K$. Then the 2-class field tower of $E=F K$ is infinite.

Proof. Since $n=4$ and $t \geq 8 \geq 3+2 \sqrt{4+1}=7.472 \ldots$ in these cases, the 2-class field tower of $E=F K$ is infinite by Martinet's inequality I.

MARTINET'S INEQUALITY II. Let $F$ be a totally imaginary number field of degree $n$, and $E$ a quadratic extension of $F$. Let $t$ be the number of prime ideals of $F$ which ramify in $E$. If

$$
t \geq n / 2+3+2 \sqrt{n+1}
$$

then the 2-class field tower of $E$ is infinite.

Proof. Since $r_{1}=\varrho=0$ and $r_{2}=n / 2$, the assertion follows from the general case of Martinet's inequality.

Corollary 3. Let $F$ be an imaginary quadratic number field. Suppose that four rational primes split in $F$ and ramify in $K$. Then the 2-class field tower of $E=F K$ is infinite.

Proof. Since $n=2$ and $t \geq 8 \geq 2 / 2+3+2 \sqrt{2+1}=7.464 \ldots$, the 2 -class field tower of $E=F K$ is infinite by Martinet's inequality II. 
3. The case with one negative prime discriminant. Let $K$ be an imaginary quadratic number field with discriminant $d$. First we recall some properties of Rédei matrices of quadratic number fields [10, 11, 13, 14].

A rational integer is called a discriminant if it is the discriminant of a quadratic number field or equal to 1 . A discriminant which is divisible by only one prime is called a prime discriminant. Prime discriminants are denoted by $p^{*}=(-1)^{(p-1) / 2} p$ (if $p$ is an odd prime), or $p^{*}=-4,8$ or -8 (if $p$ is equal to 2$)$. Let $d=p_{1}^{*} p_{2}^{*} \ldots p_{t}^{*}$ be the unique factorization of $d$ into a product of prime discriminants. By genus theory, we have 2 -rank $C_{K}=t-1$.

Using Kronecker symbols $\left(\frac{D}{p}\right)$, where $D$ is a discriminant and $p$ is a prime number satisfying $p \nmid D$, we define the Rédei matrix $R_{K}=\left(a_{i j}\right) \in$ $\mathbf{M}_{t \times t}(\mathbb{Z} / 2 \mathbb{Z})$ of $K$ by

$$
(-1)^{a_{i j}}= \begin{cases}\left(\frac{p_{i}^{*}}{p_{j}}\right) & (i \neq j), \\ \left(\frac{d / p_{i}^{*}}{p_{i}}\right) & (i=j) .\end{cases}
$$

By the definition of the Kronecker symbol, we have $a_{i j}=0(i \neq j)$ if and only if the rational prime $p_{j}$ splits in $\mathbb{Q}\left(\sqrt{p_{i}^{*}}\right)$. Note that the sum of all row vectors of $R_{K}$ is equal to the zero vector $\mathbf{0}$ in $(\mathbb{Z} / 2 \mathbb{Z})^{t}$ so that rank $R_{K} \leq t-1$ and the solution space $X$ of the linear equations $\boldsymbol{x} R_{K}=\mathbf{0}\left(\boldsymbol{x} \in(\mathbb{Z} / 2 \mathbb{Z})^{t}\right)$ contains the vector $\mathbf{1}=(1,1, \ldots, 1)$ ( $t$ times). By the results of Rédei and Rédei-Reichardt, we have 4-rank $C_{K}=t-1-\operatorname{rank} R_{K}$.

In the case where $p_{i}^{*} \neq-4$ and $p_{j}^{*} \neq-4$, we have $a_{i j}=a_{j i}(i \neq j)$ if and only if $p_{i}^{*}>0$ or $p_{j}^{*}>0$, by the quadratic reciprocity law. Therefore, if exactly one negative prime discriminant $(\neq-4)$ divides $d$, then $R_{K}$ is a symmetric matrix.

THEOREM. Let $K$ be an imaginary quadratic number field with discriminant d. Suppose that 2-rank $C_{K}=4$ and exactly one negative prime discriminant divides $d$. Let $d=p_{1}^{*} p_{2}^{*} p_{3}^{*} p_{4}^{*} p_{5}^{*}\left(p_{1}^{*}<0\right)$ be the unique factorization of $d$ into a product of prime discriminants. Then the 2-class field tower of $K$ is infinite, except the case where

$$
R_{K}=\left(\begin{array}{lllll}
0 & 1 & 1 & 1 & 1 \\
1 & 1 & 0 & 1 & 1 \\
1 & 0 & 1 & 1 & 1 \\
1 & 1 & 1 & 1 & 0 \\
1 & 1 & 1 & 0 & 1
\end{array}\right),
$$

by changing the order of $p_{i}$ 's $(2 \leq i \leq 5)$. In the exceptional case, $p_{1}^{*} \neq-4$ and the 4-rank of $C_{K}$ is equal to 0 . 
Proof. First, suppose that $p_{1}^{*}=-4$. Then we have $p_{j} \equiv 1(\bmod 4)$ for any $j(2 \leq j \leq 5)$. Put $F=\mathbb{Q}\left(\sqrt{p_{1}^{*}}\right)=\mathbb{Q}(\sqrt{-1})$. Then the four rational primes $p_{j}(2 \leq j \leq 5)$ split in $F$ and ramify in $K$. Hence, the 2-class field tower of $E=F K=K(\sqrt{-1})$ is infinite by Corollary 3. Since $E / K$ is an unramified 2-extension, the 2-class field tower of $K$ is also infinite.

In the following, we assume that $p_{1}^{*} \neq-4$. Therefore, $R_{K}$ is a symmetric matrix. For each Rédei matrix $R_{K}$, if we could find a subfield $F=$ $\mathbb{Q}\left(\sqrt{p_{i}}, \sqrt{p_{j}}\right), \mathbb{Q}\left(\sqrt{p_{i}}, \sqrt{p_{j} p_{k}}\right)$ or $\mathbb{Q}\left(\sqrt{p_{i} p_{j}}, \sqrt{p_{i} p_{k}}\right)(i, j, k \in\{2,3,4,5\})$ of the genus field $\mathbb{Q}\left(\sqrt{p_{1}^{*}}, \ldots, \sqrt{p_{5}^{*}}\right)$ of $K$ which satisfies the condition of Corollary 2, then the 2-class field tower of $E=F K$ would be infinite. Since $E / K$ is an unramified 2-extension, we conclude that the 2-class field tower of $K$ is also infinite, in those cases.

First, suppose that there exists a column vector $\boldsymbol{a}_{j}=\left(a_{i j}\right)(1 \leq j \leq 5)$ of $R_{K}$ for which at least two of $a_{i j}$ 's $(2 \leq i \leq 5, i \neq j)$ are 0 . Then, assuming that $a_{i j}=a_{k j}=0(i \neq j, k \neq j)$, we put $F=\mathbb{Q}\left(\sqrt{p_{i}}, \sqrt{p_{k}}\right)$. Since the rational prime $p_{j}$ splits completely in $F$ and ramifies in $K$, and two rational primes $p_{l}, p_{m}(\{i, j, k, l, m\}=\{1,2,3,4,5\})$ are unramified and split into at least two primes in $F$ and ramify in $K$, the 2-class field tower of $E=F K$ is infinite by Corollary 2. Hence, the 2-class field tower of $K$ is also infinite.

In the following, we assume that at most one of $a_{i j}$ 's $(2 \leq i \leq 5, i \neq j)$ is 0 for each column vector $\boldsymbol{a}_{j}=\left(a_{i j}\right)(1 \leq j \leq 5)$ of $R_{K}$.

(i) One of $a_{i 1}$ 's $(2 \leq i \leq 5)$ is 0 : In this case, we may assume that $a_{21}=0$ and $a_{31}=a_{41}=a_{51}=1$ without loss of generality. If $a_{32}=a_{42}=$ $a_{52}=1$, then we put $F=\mathbb{Q}\left(\sqrt{p_{3} p_{4}}, \sqrt{p_{3} p_{5}}\right)$. Since $\left(\frac{p_{i} p_{k}}{p_{j}}\right)=(-1)(-1)=1$ for each $j \in\{1,2\}$ and $i, k \in\{3,4,5\}$, the two rational primes $p_{1}$ and $p_{2}$ split completely in $F$ and ramify in $K$. Therefore, the 2-class field tower of $E=F K$ is infinite by Corollary 2, and the 2-class field tower of $K$ is also infinite. On the other hand, if one of $a_{i 2}$ 's $(3 \leq i \leq 5)$ is 0 , then we may assume that $a_{32}=0$ and $a_{42}=a_{52}=1$ without loss of generality. So, we have $a_{23}=0$ and $a_{43}=a_{53}=1$ by our assumption and

$$
R_{K}=\left(\begin{array}{lllll}
1 & 0 & 1 & 1 & 1 \\
0 & 0 & 0 & 1 & 1 \\
1 & 0 & 1 & 1 & 1 \\
1 & 1 & 1 & * & * \\
1 & 1 & 1 & * & *
\end{array}\right),
$$

where the asterisks " $*$ " mean 0 or 1 . We put $F=\mathbb{Q}\left(\sqrt{p_{2}}, \sqrt{p_{4} p_{5}}\right)$. Since $\left(\frac{p_{2}}{p_{j}}\right)=1$ and $\left(\frac{p_{4} p_{5}}{p_{j}}\right)=(-1)(-1)=1$ for $j \in\{1,3\}$, the two rational primes $p_{1}$ and $p_{3}$ split completely in $F$ and ramify in $K$. Therefore, the 2-class field 
tower of $E=F K$ is infinite by Corollary 2, and the 2-class field tower of $K$ is also infinite.

(ii) $a_{21}=a_{31}=a_{41}=a_{51}=1$ : If there exists a column vector $\boldsymbol{a}_{j}=$ $\left(a_{i j}\right)(2 \leq j \leq 5)$ of $R_{K}$ satisfying $a_{i j}=1$ for all $i(2 \leq i \leq 5, i \neq j)$, then we put

$$
F=\mathbb{Q}\left(\sqrt{p_{k} p_{l}}, \sqrt{p_{k} p_{m}}\right) \quad(\{j, k, l, m\}=\{2,3,4,5\}) .
$$

In this case, as in the first half of the case (i), we see that the two rational primes $p_{1}$ and $p_{j}$ split completely in $F$ and ramify in $K$. Therefore, the 2-class field tower of $E=F K$ is infinite by Corollary 2, and the 2-class field tower of $K$ is also infinite. However, if there exists no such column vector $\boldsymbol{a}_{j}(2 \leq j \leq 5)$ of $R_{K}$, then we cannot find an appropriate field $F$ which satisfies the condition of Martinet's inequality. In this case, we have $a_{23}=a_{32}=a_{45}=a_{54}=0$, by changing the order of $p_{i}$ 's. So, $R_{K}$ is as described in the assertion of our Theorem. This completes the proof of the Theorem.

REMARK 1. In Theorem 1 of [1], Benjamin classified the case with only one negative prime discriminant $(\neq-4)$ and 2-rank $C_{K}=4$ into 32 types, by using "Kronecker symbol configurations". Among them, the infinitude of the 2-class field tower remained unsettled for 5 types. Actually, there are two more Kronecker symbol configurations

$$
\left(\frac{p_{1}}{p_{3}}\right)=\left(\frac{p_{1}}{p_{4}}\right)=\left(\frac{p_{2}}{p_{3}}\right)=\left(\frac{p_{2}}{p_{4}}\right)=1
$$

with 4 -rank $C_{K}=0$, and

$$
\left(\frac{p_{1}}{p_{3}}\right)=\left(\frac{p_{1}}{p_{4}}\right)=\left(\frac{p_{2}}{p_{3}}\right)=\left(\frac{p_{2}}{p_{4}}\right)=-1
$$

with 4-rank $C_{K}=2$. The numbers of Rédei matrices (= the numbers of Kronecker symbol configurations) with given 4-rank are as follows:

\begin{tabular}{c|ccccc|c} 
4-rank $C_{K}$ & 4 & 3 & 2 & 1 & 0 & total \\
\hline \# of Rédei matrices & 1 & 2 & 8 & 10 & 13 & 34
\end{tabular}

In our Theorem, we showed the infinitude of the 2-class field tower for 33 types, except the third case of Theorem $1(\mathrm{C})$ in [1] where $p_{1}^{*}$ is negative $(\neq-4)$ and

$$
\left(\frac{p_{2}}{p_{3}}\right)=\left(\frac{p_{4}}{p_{5}}\right)=1 .
$$

ExAMPLES. The following are some examples of imaginary quadratic number fields with only one negative prime discriminant $(\neq-4), 2$-rank $C_{K}$ 
$=4$ and the Rédei matrix of exceptional type:

$$
\begin{array}{ll}
\mathbb{Q}(\sqrt{-3 \cdot 5 \cdot 29 \cdot 8 \cdot 17}), & \mathbb{Q}(\sqrt{-7 \cdot 5 \cdot 41 \cdot 13 \cdot 17}), \\
\mathbb{Q}(\sqrt{-3 \cdot 5 \cdot 41 \cdot 17 \cdot 53}), & \mathbb{Q}(\sqrt{-8 \cdot 5 \cdot 61 \cdot 37 \cdot 53}) .
\end{array}
$$

REMARK 2. In Theorems 1 and 2 of [2], Benjamin proved the infinitude of the 2-class field tower of an imaginary quadratic number field $K$ with 2-rank $C_{K}=4$ and 4-rank $C_{K}=2$, in the case where $R_{K}$ is not of the type

$$
\left(\begin{array}{lllll}
* & 1 & 1 & 0 & 0 \\
* & 1 & 1 & 1 & 1 \\
* & 0 & 0 & 1 & 1 \\
1 & 1 & 1 & * & * \\
1 & 1 & 1 & * & *
\end{array}\right)
$$

with

$$
p_{1}^{*}=-4, \quad p_{2}^{*}<0, \quad p_{3}^{*}<0, \quad p_{4}^{*}>0, \quad p_{5}^{*}>0 .
$$

With the methods above one can prove the theorems of Benjamin and KochHajir as well.

Acknowledgments. The author would like to thank the referee for his careful reading of the manuscript and for his helpful comments and suggestions.

\section{References}

[1] E. Benjamin, On imaginary quadratic number fields with 2-class group of rank 4 and infinite 2-class field tower, Pacific J. Math. 201 (2001), 257-266.

[2] -, On a question of Martinet concerning the 2-class field tower of imaginary quadratic number fields, Ann. Sci. Math. Québec 26 (2002), 1-13.

[3] E. S. Golod and I. R. Shafarevich, On class field towers, Izv. Akad. Nauk SSSR Ser. Mat. 28 (1964), 261-272; English transl.: Amer. Math. Soc. Transl. (2) 48 (1965), 91-102.

[4] F. Hajir, On a theorem of Koch, Pacific J. Math. 176 (1996), 15-18.

[5] -, Correction to "On a theorem of Koch", ibid. 196 (2000), 507-508.

[6] H. Koch, Zum Satz von Golod-Schafarewitsch, Math. Nachr. 42 (1969), 321-333.

[7] F. Lemmermeyer, On 2-class field towers of some imaginary quadratic number fields, Abh. Math. Sem. Univ. Hamburg 67 (1997), 205-214.

[8] C. Maire, Un raffinement du théorème de Golod-Safarevic, Nagoya Math. J. 150 (1998), 1-11.

[9] J. Martinet, Tours de corps de classes et estimations de discriminants, Invent. Math. 44 (1978), 65-73.

[10] L. Rédei, Arithmetischer Beweis des Satzes über die Anzahl der durch vier teilbaren Invarianten der absoluten Klassengruppe im quadratischen Zahlkörper, J. Reine Angew. Math. 171 (1934), 55-60. 
[11] L. Rédei und H. Reichardt, Die Anzahl der durch 4 teilbaren Invarianten der Klassengruppe eines beliebigen quadratischen Zahlkörpers, ibid. 170 (1933), 69-74.

[12] P. Roquette, On class field towers, in: Algebraic Number Theory, J. W. S. Cassels and A. Fröhlich (eds.), Academic Press, 1967, 231-249.

[13] Y. Sueyoshi, On a comparison of the 4-ranks of the narrow ideal class groups of $\mathbb{Q}(\sqrt{m})$ and $\mathbb{Q}(\sqrt{-m})$, Kyushu J. Math. 51 (1997), 261-272.

[14] —, On Rédei matrices with minimal rank, Far East J. Math. Sci. 3 (2001), 121-128.

[15] E. B. Vinberg, On the dimension theorem of associative algebras, Izv. Akad. Nauk SSSR Ser. Mat. 29 (1965), 209-214 (in Russian).

Department of Computer and Information Sciences

Faculty of Engineering

Nagasaki University

1-14 Bunkyo-machi

Nagasaki 852-8521, Japan

E-mail: sueyoshi@net.nagasaki-u.ac.jp

Received on 12.6.2003

and in revised form on 18.9.2003 